\title{
EARLY STAR FORMATION AND THE EVOLUTION OF THE STELLAR INITIAL MASS FUNCTION IN GALAXIES
}

\author{
Richard B. Larson \\ Yale Astronomy Department, Box 208101, New Haven, CT 06520-8101, USA \\ larson@astro.yale.edu
}

\begin{abstract}
It has frequently been suggested in the literature that the stellar IMF in galaxies was top-heavy at early times. This would be plausible physically if the IMF depends on a mass scale such as the Jeans mass that was higher at earlier times because of the generally higher temperatures that were present then. In this paper it is suggested, on the basis of current evidence and theory, that the IMF has a universal Salpeter-like form at the upper end but flattens below a characteristic stellar mass that may vary with time. Much of the evidence that has been attributed to a top-heavy early IMF, including the ubiquitous G-dwarf problem, the high abundance of heavy elements in clusters of galaxies, and the high rate of formation of massive stars in high-redshift galaxies, can be accounted for with such an IMF if the characteristic stellar mass was several times higher during the early stages of galaxy evolution. However, significant variations in the mass-to-light ratios of galaxies and large amounts of dark matter in stellar remnants are not as easily explained in this way because they require more extreme and less plausible assumptions about the form and variability of the IMF. Metal-free 'population III' stars are predicted to have an IMF that consists exclusively of massive stars, and they could help to account for some of the evidence that has been attributed to a top-heavy early IMF, as well as contributing importantly to the energetics and chemical enrichment of the early universe.
\end{abstract}

\section{INTRODUCTION}

The stellar initial mass function (IMF), or distribution of masses with which stars are formed, is of fundamental importance in determining the properties of stellar systems and how they evolve with time; even the processes of galaxy formation cannot be adequately understood without a knowledge of the IMF at early times. Efforts to model the formation and evolution of galaxies have generally assumed that the IMF is a universal function that does not vary with time, yet it has long been recognized that many of the predictions of such models would be altered importantly if the IMF were to vary. This is because the present luminosities of galaxies depend mostly on the number of stars they contain with masses near one solar mass, but their heavy-element contents and the energy feedback effects that control the evolution of their gas contents depend on the number of stars that formed in them with masses above ten solar masses; thus, if the relative numbers of low-mass and high-mass stars produced had been different at early times, such basic properties of galaxies as their luminosities, colors, and chemical compositions and their evolution with time 
would be substantially altered. This would have far-reaching implications for the interpretation of many observations of galaxies, especially observations at high redshifts.

For many years, beginning with Schwarzschild \& Spitzer (1953), there have been speculations in the literature that the IMF was dominated by massive stars at early times. Some of the evidence for a variable IMF, and some of the possible implications of models of galaxy evolution with an IMF that was more top-heavy at early times, were discussed earlier by Larson (1986a,b, hereafter L86). Although it now appears that the specific model proposed by L86 was probably too extreme (Meusinger 1989; Larson 1992b), a number of lines of evidence have continued to suggest that the IMF in galaxies may have been biased toward massive stars at early times. The following types of evidence will be discussed in some detail in this paper, along with their possible interpretation in terms of a time-varying IMF:

(1) The original reason for proposing that the early IMF was dominated by massive stars was that standard hot-big-bang cosmology predicts that the first stars contained no heavy elements, yet no metal-free stars have ever been found. Although it is possible that the number of such stars formed was negligible, this is not very plausible because a non-negligible amount of time must have elapsed between the formation of the first metal-free stars and the appearance of the first stars polluted with their nucleosynthetic products.

(2) A similar problem of long standing is the classical 'G-dwarf problem', namely the deficiency of metal-poor stars in the solar neighborhood compared with the predictions of simple models of chemical evolution. A related fact is the observation that the abundances of heavy elements in the Galactic disk have shown only a slow increase with time over most of Galactic history. These observations suggest that the rate of production of heavy elements was higher at early times than at more recent times.

(3) The large total mass of heavy elements observed in the hot gas in large clusters of galaxies suggests that the early IMF in these systems contained a higher proportion of massive stars than the present-day IMF. The increase of both the metallicities and the mass-to-light ratios of cluster galaxies with mass also suggests that the IMF may have been more top-heavy in the most massive galaxies, which could have been the first to form their stars.

(4) There is possible evidence that the gas in clusters of galaxies was heated by more early supernovae than would have been predicted by a standard IMF, and this again suggests a top-heavy early IMF in clusters.

(5) Recent studies of galaxy populations at high redshifts have suggested that a top-heavy early IMF may be needed to account for the strong evolution of the cosmic luminosity density with redshift, especially if there is significant dust extinction in high-redshift galaxies.

(6) Standard hot-big-bang cosmology suggests that most of the baryonic matter in the universe is in a dark form, and one possibility could be the dark remnants of early generations of massive stars. The claimed detection via microlensing of stellar-mass 'machos' in the halo of our Galaxy has led to the suggestion that a significant amount of dark matter might be in stellar remnants.

Most of the models that have been developed to describe the formation and evolution of galaxies have assumed that the stellar IMF is a power law similar to that originally proposed by Salpeter (1955); if any variability of the IMF has been allowed, this variability has usually been assumed to take the form of a change in the slope of the power law. However, this assumption is not supported by recent evidence suggesting that the IMF in many nearby systems always has a slope similar to the Salpeter value above a solar mass, while it flattens at lower masses, possibly even turning down below a few tenths of a solar mass. The departure of the IMF from a power law at the low end might reflect the existence of a mass scale in the star formation process that depends on cloud properties such as temperature and pressure, and such a mass scale might be expected to vary with place and time. In this case, another possible form for a varying IMF might be given by 
a function that has a universal power-law form at large masses but departs from this power law below a characteristic mass that can vary. The mass scale for star formation probably depends most strongly on temperature, and since the temperature in star-forming clouds was almost certainly higher at earlier cosmic times, the mass scale should also have been higher at earlier times. This would have the effect of decreasing the relative number of low-mass stars formed at early times.

In this paper it will be shown that many of the observations mentioned above can be accounted for with an IMF whose mass scale was higher at early times, and the constraints that can be placed on such models will be discussed. Section 2 summarizes current evidence and theory regarding the present-day IMF, and Section 3 discusses how the associated mass scale might be expected to vary with time. Section 4 considers whether such a time-varying IMF can account for the chemical evidence mentioned in items (1)-(3) above and the thermal evidence mentioned in item (4). Section 5 discusses the possible evidence for variability of the IMF provided by high-redshift observations, and Section 6 discusses the extent to which models with a top-heavy early IMF might account for dark matter and machos. Finally, Section 7 discusses the possibility that metal-free 'population III' stars with an IMF strongly biased toward high masses might have existed in significant numbers and might help to account for some of the observations mentioned above. The conclusions of the paper are summarized in Section 8.

\section{EVIDENCE AND THEORY CONCERNING THE PRESENT-DAY IMF}

As has been known since the work of Salpeter (1955), the present-day IMF of stars in the solar neighborhood can be approximated by a declining power law for masses above a solar mass, but it falls below an extrapolation of this power law at lower masses, flattening below about $0.5 \mathrm{M}_{\odot}$ and possibly even declining (in number of stars per unit logarithmic mass interval) below $0.25 \mathrm{M}_{\odot}$ (Scalo 1986, 1998). The behavior of the IMF at the lowest masses remains very uncertain because of the poorly known mass-luminosity relation for the faintest stars; most studies have suggested that the IMF is roughly flat (in logarithmic mass units) at the low end, but a significant falloff below $0.1 \mathrm{M}_{\odot}$ seems indicated by the relative paucity of brown dwarfs (Basri \& Marcy 1997). The IMF in open clusters is generally similar to that of the field stars, although possibly more variable; the best-studied young cluster, the Orion Nebula cluster, has an IMF very similar to that derived by Scalo (1986) for field stars, including a decline below $0.2 \mathrm{M}_{\odot}$ (Hillenbrand 1997). Thus, present evidence allows the possibility that the IMF in the solar neighborhood may be roughly flat at the low end, or that it may peak at a mass of around $0.25 \mathrm{M}_{\odot}$ and decline into the brown-dwarf regime, or even that it may be quite variable at the low end. Whether or not there is a peak, the flattening of the IMF below a solar mass implies that there is a characteristic stellar mass of the order of one solar mass such that half of the mass that condenses into stars goes into stars above this mass and half goes into less massive stars.

Recent studies of the IMF in clusters and associations in our Galaxy and the Magellanic Clouds have generally supported the universality of the power-law part of the IMF above $1 \mathrm{M}_{\odot}$, and have shown that in most cases the slope $x$, defined such that $d N / d \log m \propto m^{-x}$, is not very different from the original Salpeter value $x=1.35$ (von Hippel et al. 1996; Hunter et al. 1997; Massey 1998). However, Scalo (1998) emphasizes the large scatter that still exists in these results, and notes possible trends with mass such that the slope at intermediate masses may be significantly steeper than the Salpeter value and equal to $x \sim 1.7 \pm 0.5$, similar to what was found earlier by Scalo (1986). Massey (1998) also finds evidence that the slope is steeper for field stars than for clusters and associations. Nevertheless, clusters and associations probably yield more reliable results than field stars because fewer assumptions are required to derive their IMFs, and the Salpeter slope is representative of the results found for them at masses above a solar mass, as is illustrated in Figure 5 of Scalo (1998). At lower masses the IMF slope clearly becomes much smaller than the Salpeter value, and estimates of the slope below 0.5 solar masses generally fall in 
the range $x \sim 0 \pm 0.5$. The IMF below $0.1 \mathrm{M}_{\odot}$ remains very poorly known because only a small number of objects have yet been found in this mass range, and suggested values of the slope in the brown-dwarf regime range from $x \sim-1$, implying an IMF that declines significantly at the low end, to $x \sim 0$, implying an IMF that is flat at the low end (e.g., Basri \& Marcy 1997; Martín et al. 1998; Mayor, Queloz \& Udry 1998; Festin 1998).

Since the actual IMF thus remains quite uncertain, especially at the low end, we shall consider two simple approximations that encompass the range of possibilities suggested by the evidence mentioned above. If brown dwarfs are indeed rare objects and the IMF peaks at mass of a few tenths of a solar mass, its shape may be similar to that of the analytic function adopted by L86 to represent a low-mass mode of star formation, $d N / d \log m \propto m^{-2} \exp \left[-\left(m_{1} / m\right)^{1.5}\right]$, except for being less sharply peaked than this function and falling off more gradually toward both lower and higher masses. We therefore consider, as one possibility, the following simple analytic form:

$$
d N / d \log m \propto m^{-1.35} \exp \left(-m_{1} / m\right) .
$$

This function has a logarithmic slope $x=1.35-m_{1} / m$, so it approaches a power law with the Salpeter slope $x=1.35$ at large masses, peaks at a mass $m_{\mathrm{p}}=m_{1} / 1.35$, and falls off exponentially with increasingly negative $x$ at lower masses. Since this function has a steeper falloff at the low end than is suggested by most of the evidence mentioned above, we consider also the possibility that the IMF does not decline at all at the low end. If brown dwarfs are as common as is suggested by the most optimistic recent estimates, and if the IMF accordingly is approximately flat at the low end, it may be represented approximately by the following simple alternative form:

$$
d N / d \log m \propto\left(1+m / m_{1}\right)^{-1.35} .
$$

This function is very similar to (1) at masses above $m_{1}$ and has a logarithmic slope $x=1.35(1+$ $\left.m_{1} / m\right)^{-1}$, so that it again approaches the Salpeter form at large masses but becomes asymptotically flat with $x=0$ at the low end. Approximations (1) and (2) are thus consistent with the evidence summarized above that the IMF typically has a Salpeter form for large masses, while they allow for a large range of uncertainty or variability of the lower IMF, and they also allow for the possibility that the mass scale $m_{1}$ may be variable.

The mass scale $m_{1}$ might be expected to be related to a fundamental scale in the star formation process such as the Jeans mass, and evidence supporting this possibility has been discussed by Larson $(1995,1996,1998)$. Analyses of the spatial clustering of the T Tauri stars in the Taurus and Ophiuchus regions have revealed the existence of two distinct regimes, a hierarchical clustering regime on large scales and a binary regime on small scales, with a break occurring at a separation of about $0.05 \mathrm{pc}$ in a plot of average companion surface density versus separation (Larson 1995; Simon 1997). The existence of this break suggests that the clustering hierarchy is built up of basic star-forming units with a radius of $\sim 0.05 \mathrm{pc}$ that typically form binary stars. These units can be identified with the 'ammonia cores' in which $\mathrm{T}$ Tauri stars form, and these cores have masses of the order of $1 \mathrm{M}_{\odot}$, consistent with the possibility that they typically form two stars with masses of $\sim 0.5 \mathrm{M}_{\odot}$. These radii and masses are very similar to the radius and mass of a marginally stable Bonnor-Ebert sphere with a temperature of $10 \mathrm{~K}$ and a boundary pressure equal to the typical non-thermal pressure of $\sim 3 \times 10^{5} \mathrm{~cm}^{-3} \mathrm{~K}$ observed in molecular clouds (Larson 1996, 1998). The Jeans scale calculated in this way can be regarded as the scale at which there is a transition between a chaotic regime dominated by non-thermal pressures on larger scales and a regular regime dominated by thermal pressure on smaller scales; whatever the details of the star formation process, this transition scale almost certainly plays some role in determining stellar masses and the IMF.

Direct evidence for a transition between different physical regimes on different scales has been provided by studies of the internal kinematics of star-forming cloud cores by Goodman et al. (1998). On scales larger than about $0.1 \mathrm{pc}$, the non-thermal component of the velocity dispersion 
in these cores increases with region size following the general linewidth-size relation for molecular clouds, while on smaller scales the non-thermal velocity dispersion becomes smaller than the sound speed and almost independent of region size. Goodman et al. interpret these results as indicating a transition from a regime of chaotic motion on larger scales to a regime of 'velocity coherence' on smaller scales, and they note that this transition occurs at a scale similar to that found for the break in the clustering properties of $\mathrm{T}$ Tauri stars mentioned above and suggest that both results reflect the existence of an 'inner scale' of a self-similar process, i.e. a scale at which there is a transition from chaotic behavior on larger scales to regular behavior on smaller scales. If the physical basis of this transition is the transition from dominance by non-thermal pressures on large scales to dominance by thermal pressure on small scales, the associated scale is just the Jeans scale as defined above. Thus, both theoretical and observational considerations suggest that there is an intrinsic scale in the star formation process that can be identified with the Jeans scale. The power-law upper part of the IMF may be produced by self-similar accumulation processes occurring in the chaotic regime, possibly arising from the universal scale-free dynamics of turbulence and possibly describable by fractal geometry (Larson 1992a, 1995; Elmegreen 1997).

The Jeans mass depends on the temperature and pressure in star-forming clouds and is proportional to $T^{2} P^{-1 / 2}$. In self-gravitating clouds the pressure is proportional to the square of the surface density $\mu$, and the Jeans mass is therefore proportional to $T^{2} \mu^{-1}$, a result also found from stability analyses of self-gravitating flattened or filamentary structures (Larson 1985). Because of the strong dependence of this mass scale on the temperature, it seems likely that the temperature in star-forming clouds is the most important parameter controlling the mass scale for star formation (Larson 1985), although the effect of changes in temperature can be compensated by sufficiently large changes in pressure or surface density. The temperature is controlled by the balance between heating by external radiation fields and cooling by line emission from atoms and molecules and thermal emission from dust, while the pressure is controlled by the dynamical processes that create and confine star-forming clouds (Larson 1996). More intense external radiation fields and lower heavy-element abundances would both imply higher cloud temperatures, and thus would increase the mass scale for star formation unless there is a sufficiently large compensating increase in pressure. These effects provide a basis for order-of-magnitude estimates of how the IMF might vary with time or metallicity in galaxies, as will be discussed in the next section. Note that a strong dependence of the mass scale for star formation on cloud temperature is expected on quite general grounds; for example, it is also predicted by the hypothesis that stellar masses are controlled by a temperature-dependent accretion rate (Adams \& Laughlin 1996).

\section{POSSIBLE TIME VARIABILITY OF THE IMF}

Past studies exploring the effect of a time-varying IMF have mostly assumed either a power-law IMF with a variable slope (Schmidt 1963) or a bimodal IMF with high-mass and low-mass components that can vary independently (L86). However, as has been noted above, present evidence does not support either a variable slope or the existence of bimodality or any other kind of 'fine structure' in the IMF; in particular, the kink near $1 \mathrm{M}_{\odot}$ that was considered by Scalo (1986) and L86 to provide possible evidence for bimodality now seems better interpreted as an artifact resulting from an inaccurate mass-luminosity relation. The evidence and theory summarized above suggest instead that the IMF may have a universal slope at the upper end, and that any variability may be confined to the lower end, possibly being associated with a variable mass scale $m_{1}$ at which it departs from a power law. The essential effect of this type of variability is to alter the relative number of low-mass stars formed, compared with the number of more massive stars; if the mass scale was higher at earlier times, relatively fewer low-mass stars would have been formed at these times. 
It was suggested above that the mass scale of the IMF depends mainly on the temperature in star-forming clouds. This temperature was almost certainly higher at earlier times for several reasons: (1) The cosmic background temperature, which is the minimum possible cloud temperature, increases with redshift $z$ as $T=2.73(1+z)$, and it becomes higher than the present minimum temperature of $\sim 8 \mathrm{~K}$ in molecular clouds at redshifts greater than 1.9. (2) The generally lower metallicity of the gas at early times implies lower cooling rates, and hence higher temperatures for a given heating rate. (3) The heating rate was almost certainly higher at early times because of the more intense radiation fields that were present at all wavelengths; in particular, ultraviolet radiation from young stars and cosmic rays from supernovae, which are the most important heating effects at the present time, would both have been more important at early times when the star formation rate per unit volume was higher. (4) Starburst activity, which may produce a locally top-heavy IMF for similar reasons, may have been more prevalent during the early stages of galactic evolution, especially if mergers played an important role.

Since the Jeans mass depends on the pressure as well as the temperature of star-forming clouds, the effect of higher temperatures could have been compensated by much higher pressures. However, there is no apparent reason why the pressures in star-forming clouds should have been much higher at earlier times. At the present time, the typical pressure in star-forming molecular clouds is already much higher than the general pressure of the interstellar medium, probably as a result of the thermal and dynamical feedback effects of star formation on the ISM (Larson 1996). At early times, the feedback effects that pressurize present-day molecular clouds might not yet have become established, and such large pressure enhancements might not have been common. The extreme pressures needed to form low-mass stars at the higher temperatures present at early times might have existed only in exceptionally dense and massive clouds, such as those that may have formed the globular clusters. If so, the formation of low-mass stars at early times might have occurred only in relatively extreme circumstances, and globular clusters might not be representative of early star formation.

Thus, while it seems likely that the IMF was generally more top-heavy at earlier times, many physical effects were probably involved and no quantitative predictions can yet be made. However, it is worth noting that even the minimal effect of a higher cosmic background temperature can significantly increase the Jeans mass during early stages of galaxy evolution. Even if the cloud temperature increases by the minimum possible amount with redshift and is equal to either the present $8 \mathrm{~K}$ or the background temperature $2.73(1+z)$ when the latter is higher, the predicted Jeans mass for a given pressure increases by a factor of 2 at a redshift of 3, and by a factor of 4 at a redshift of 5 . If the minimum cloud temperature is twice the background temperature at the higher redshifts, the Jeans mass for a given pressure increases by a factor of 7 at a redshift of 3 and a factor of 17 at a redshift of 5 . Even though the possible compensating effect of a higher pressure is difficult to estimate, these examples suggest that the mass scale of the IMF could plausibly have been several times higher during the early stages of galaxy evolution, and higher by an order of magnitude or more at redshifts larger than 5.

To illustrate the possible consequences of such an increase with redshift in the mass scale of the IMF, if the peak mass $m_{\mathrm{p}}$ in IMF (1) is increased by a factor of 4 from 0.25 to $1.0 \mathrm{M}_{\odot}$, the ratio of the number of solar-mass stars formed to the number of very massive stars formed is decreased by a factor of 2.8; for IMF (2), which is flatter at the low end, this ratio is decreased by a factor of 2.1 for the corresponding increase in the mass scale $m_{1}$. If the mass scale of the IMF is increased by a further factor of 2, the ratio of solar-mass stars to very massive stars is decreased by a factor of 11 for IMF (1) and a factor of 4 for IMF (2). Such changes are large enough to have very important consequences for galactic evolution, as will be discussed further below.

Is there any direct evidence that the IMF has varied with time in our Galaxy? Globular clusters have been extensively studied to look for evidence of variability of the IMF, but it is now recognized that the effects of dynamical evolution are very important for these systems and that this makes it difficult to derive their initial mass functions. In any case, globular clusters might 
not be representative of all early star formation, as was noted above, so a better test of variability of the IMF with time might be to compare the IMF of halo field stars with that of disk stars. The Galactic halo has a flatter luminosity function than the disk (Dahn et al. 1995; Reid et al. 1996; Méndez et al. 1996), but this does not necessarily imply a flatter mass function because of the different mass-luminosity relations for metal-poor and metal-rich stars. When this is taken into account, any evidence that the halo has a flatter mass function than the disk becomes marginal, and all that can be said with confidence is that the halo IMF is not unusually steep. For example, Richer \& Fahlman (1997) and Chabrier \& Méra (1997) find that the slope of the IMF for halo stars is approximately $x \sim 0.6$ to 0.8 , and although this is smaller than the Salpeter slope $x=1.35$, it is not significantly smaller than the slope $x \sim 0.9$ characteristic of the disk IMF in the same mass range below a solar mass.

Thus there is at present no clear direct evidence that the IMF in our Galaxy has varied with time. The best evidence for a variable IMF may therefore be provided by the observed chemical abundances in the stars in our Galaxy and others, and especially by the high heavy-element abundances in the hot gas in clusters, as will be discussed in the following section. Additional evidence may also be provided by recent observations suggesting high rates of massive star formation in galaxies at high redshifts, as will be discussed in Section 5 .

\section{EVIDENCE FROM CHEMICAL ABUNDANCES AND SUPERNOVA RATES}

In this section we consider the evidence from chemical abundances suggesting that the IMF has varied with time in our Galaxy and others, and we address the question of whether this evidence can be accounted for with an IMF such as (1) or (2) in which the mass scale $m_{1}$ has varied with time. The types of evidence considered here include (1) the classical G-dwarf problem and the related slow variation of metallicity with time over most of Galactic history, (2) the chemical abundances observed in elliptical galaxies and the hot gas in clusters of galaxies, and (3) the energetics of the hot gas in clusters.

\subsection{Chemical evolution of galaxies and the G-dwarf problem}

The paucity of metal-poor stars in the solar neighborhood relative to the predictions of simple models of chemical evolution was noted by van den Bergh (1962) and discussed further by Schmidt (1963), who suggested that this problem, the classical 'G-dwarf problem', can be solved if the IMF has varied with time in such a way that relatively more massive stars were formed at earlier times. A related observation is the fact that the average metallicity of nearby stars has shown only a modest increase with time over most of Galactic history, and this relatively flat 'age-metallicity relation' can also be accounted for with a time-varying IMF; for example, it is reproduced by the bimodal-IMF model of L86 in which a high-mass mode of star formation dominates at early times. However, nearly all recent efforts to model the chemical evolution of galaxies have assumed instead that the G-dwarf problem and the flat age-metallicity relation are explained by the continuing infall of metal-free primordial gas (Larson 1972); most authors have regarded a variable IMF as an ad hoc hypothesis, while infall has been considered a more natural solution to the problem because some amount of continuing infall is predicted by many models of galaxy formation. It is therefore relevant to consider again whether infall is really a satisfactory solution to the G-dwarf problem.

The original suggestions by Oort (1970) and Larson (1972) that continuing infall is important for galactic evolution were based on the assumption that galaxy formation is an inefficient process and leaves behind a large reservoir of primordial gas that continues be accreted by galaxies for a long period of time. However, it now appears that most of the known intergalactic gas has been heated and enriched in heavy elements by the effects of early star formation in galaxies; this is true at least in the large clusters of galaxies that are considered to be representative samples of 
the universe. Thus, there may no longer be much truly primordial gas, and indeed little evidence has ever been found for such gas despite many years of searching. The halo of our Galaxy contains some 'high-velocity clouds' that might represent infalling (but not primordial) material, yet the total mass in these clouds is only about $10^{7} \mathrm{M}_{\odot}$, and their origin remains unknown (Wakker \& van Woerden 1997). Even if they do represent infalling gas, the estimated infall rate is only a few tenths of a solar mass per year (Mirabel 1989), an order of magnitude too small to be of major importance for Galactic chemical evolution. Infall rates may well have been much higher at early times, and this could help to solve the G-dwarf problem, but infall models require large amounts of continuing infall if they are to account for the flat age-metallicity relation, especially that indicated by observations of open clusters (Friel 1995). It is therefore questionable whether infall can by itself adequately solve the G-dwarf problem and the related flat age-metallicity relation. It may even be that most galaxies, instead of continuing to accrete primordial gas, expel significant amounts of supernova-heated and enriched gas early in their evolution (Section 4.2).

Another problem for infall models is that the G-dwarf problem has recently been found to exist also in other galaxies, including ellipticals (Bressan, Chiosi \& Fagotto 1994; Vazdekis et al. 1996; Worthey, Dorman \& Jones 1996). Bressan et al. (1994) suggest that the problem can be solved with infall models, but Vazdekis et al. (1996) and Worthey et al. (1996) consider infall models to be unlikely for elliptical galaxies and suggest instead that a variable IMF may account for the observations. There is less time for infall to be effective in elliptical galaxies than in spirals, and in any case there is evidence that the elliptical galaxies in clusters lose rather than accrete large amounts of gas (Section 4.2). One might also expect the importance of infall effects to depend on the environment, yet the G-dwarf problem seems to exist in all galaxies studied, independently of their environments; this suggests that the origin of the G-dwarf problem may be an intrinsic feature of galactic evolution such as a time-varying IMF.

A time-varying IMF can solve the G-dwarf problem if relatively few low-mass stars were formed during the early period when the metallicity was low. Since simple models of chemical evolution are based on the instantaneous recycling approximation, which applies best to elements like oxygen that are produced only in massive stars, it is best to use oxygen abundances when comparing predicted and observed abundance distributions. Studies of the oxygen abundance distribution of nearby stars have consistently shown that only about $5-10 \%$ of the stars in the solar neighborhood have oxygen abundances below half of the median value (Pagel 1989; Sommer-Larsen 1991; Wyse \& Gilmore 1995; Rocha-Pinto \& Maciel 1996), representing a deficiency by a factor of 3 or more relative to the simple-model prediction that $28 \%$ of the stars should have abundances in this range. These studies give the abundance distribution of all stars in a vertical column through the local Galactic disk, so they show that even at large distances from the Galactic plane there are not enough oxygen-poor stars to be consistent with a simple model. The exact magnitude of the deficiency depends somewhat on the assumed relation between oxygen abundance and iron abundance; for example, if the usual assumption that $[\mathrm{O} / \mathrm{H}]=0.5[\mathrm{Fe} / \mathrm{H}]$ is adopted, the fraction of stars in a local column having oxygen abundances below half of the median value is about $6 \%$ according to Wyse \& Gilmore (1995) and 4\% according to Rocha-Pinto \& Maciel (1996), while if it is assumed instead that $[\mathrm{O} / \mathrm{H}]=0.65[\mathrm{Fe} / \mathrm{H}]$, as may be more consistent with recent evidence (McWilliam 1997), these numbers become $12 \%$ and $9 \%$, respectively.

If we assume, accordingly, that only $10 \%$ or less of the stars in a local column have oxygen abundances below half of the median value, this can be explained if the number of low-mass stars that formed when the oxygen abundance was this low was reduced by a factor of 3 relative to the number of massive oxygen-producing stars formed. With an IMF of form (1), such a reduction can be achieved if the peak mass $m_{\mathrm{p}}$ was increased by a factor of 4 to $1 \mathrm{M}_{\odot}$ during the early oxygen-poor period; the number of $0.9 \mathrm{M}_{\odot}$ stars that formed during this period is then reduced by a factor of 2.9 relative to the number of $20 \mathrm{M}_{\odot}$ stars formed, the reduction factor being larger for stars less massive than $0.9 \mathrm{M}_{\odot}$. With an IMF of form $(2)$, the same reduction in the ratio of $0.9 \mathrm{M}_{\odot}$ stars to $20 \mathrm{M}_{\odot}$ stars is obtained if the mass scale $m_{1}$ was increased by a factor of 6 at early times. 
Since in this simple model only $10 \%$ or less of the presently observed low-mass stars were formed with a top-heavy IMF, the effect of this change in the IMF on the present luminosity function of nearby stars will be small.

The above discussion assumes that the amount of oxygen produced by massive stars is independent of their metallicity, but this may not be true because massive stars lose much of their mass in winds driven by radiation pressure, and this can cause the amount of oxygen produced to decrease with increasing metallicity (Maeder 1992). A decrease in oxygen yield with increasing metallicity might help to solve the G-dwarf problem, but the effect estimated by Maeder is less than a factor of 2 , and there is in any case a compensating effect with the opposite sign, namely that more oxygen can end up in black holes in stars of lower metallicity (Woosley \& Weaver 1995). As presently estimated, these effects do not appear to be large enough to have an important impact on the G-dwarf problem, but they probably make any quantitative prediction of oxygen yields uncertain by at least a factor of 2 .

Are models with a time-varying IMF consistent with other constraints? A general prediction of variable-IMF models is that they predict more mass in stellar remnants, mostly white dwarfs, than do standard models. If the mass of a stellar remnant is the larger of $0.6 \mathrm{M}_{\odot}$ and 0.15 times the initial stellar mass, as seems consistent with present evidence, the model of Schmidt (1963) predicts that the surface density of mass in remnants in the local Galactic disk is about $50 \mathrm{M}_{\odot} \mathrm{pc}^{-2}$, while the model of L86 predicts a surface density of about $30 \mathrm{M}_{\odot} \mathrm{pc}^{-2}$ in remnants and the above simple twostage model predicts about $12 \mathrm{M}_{\odot} \mathrm{pc}^{-2}$ in remnants. The model of Schmidt (1963) and probably the model of L86 are therefore now excluded by the fact that the dynamically determined total surface density of matter within a kiloparsec of the Galactic midplane, including halo dark matter, is only about $70 \mathrm{M}_{\odot} \mathrm{pc}^{-2}$, about $40 \mathrm{M}_{\odot} \mathrm{pc}^{-2}$ of which is already accounted for by visible stars and gas (Kuijken \& Gilmore 1991). However the above simple model assuming only the minimum change in the IMF needed to solve the G-dwarf problem is not excluded by this constraint, and in fact it does not differ much from a standard model in this respect, predicting only about $20 \%$ more mass in remnants. Thus a variable-IMF model that solves the G-dwarf problem does not necessarily predict much more mass in remnants than a standard model, and is not excluded on this basis.

Another constraint may be provided by the oxygen production rate needed to achieve agreement with observed oxygen abundances. In the above simple model, the total amount of oxygen produced for each presently visible $0.9 \mathrm{M}_{\odot}$ star is about $23 \%$ larger than in a standard model. However, this difference is much smaller than the uncertainty in the predicted oxygen yields for massive stars (Gibson 1997), so it does not significantly constrain such a model. The more extreme bimodal-IMF model of L86 overproduces oxygen by about a factor of 3 compared with a standard model, and this may exclude that model. However, it should be noted that stellar abundances do not constrain the IMF at all if galaxies lose large amounts of enriched gas to the intergalactic medium, as has apparently happened in clusters of galaxies (see below). The only firm constraint on an early top-heavy IMF is then that provided by the mass left behind in stellar remnants.

We conclude that a time-varying IMF can solve the G-dwarf problem without requiring large departures from a standard model in other respects, and without violating any of the constraints that have been discussed. The main possible problem with models assuming a time-varying IMF is that there is no clear evidence that the IMF of the oldest stars in our Galaxy, those in the halo, differs significantly from that of the disk; therefore, if the G-dwarf problem is to be solved with a time-varying IMF, either early star formation must have produced a more top-heavy IMF in the disk than in the halo, or the Galactic disk must have been pre-enriched by a population of massive stars unrelated to the visible halo population. An early epoch of preferentially massive star formation could have coincided with the period when the Galactic thick disk was formed, since the thick disk contains about $10 \%$ of the stars in a local column; at present there are no constraints on the IMF of the thick disk. The possibility that metal-free 'population III' stars could have pre-enriched the Galactic disk (Truran \& Cameron 1971) will be discussed in Section 7. 
Meanwhile, we note that it remains true that none of the explanations that has been proposed for the G-dwarf problem, including gas flows and inhomogeneous chemical evolution, can yet be definitely ruled out, and that all of them could still play some role in its solution (Tinsley 1980).

\subsection{Abundances in elliptical galaxies and the hot gas in clusters}

Since elliptical galaxies are generally older in their stellar content than spirals, they might be expected to show more evidence for a variable IMF. The observed properties of elliptical galaxies do, in fact, show several trends that might reflect a varying IMF. One such trend is the 'fundamental plane' correlation among the effective radius, surface brightness, and velocity dispersion of elliptical galaxies, which implies a systematic increase of mass-to-light ratio with mass (Guzmán, Lucey \& Bower 1993; Jørgensen, Franx \& Kjaergaard 1996). Similar trends are found quite generally in early-type galaxies (Burstein et al. 1997). In addition, both the magnesium abundance $\mathrm{Mg} / \mathrm{H}$ and the ratio of magnesium to iron abundances $\mathrm{Mg} / \mathrm{Fe}$ increase systematically with mass among early-type galaxies (Faber 1977; Worthey, Faber \& González 1992). All of these trends could in principle be caused by a varying IMF, but the increase in $\mathrm{Mg} / \mathrm{Fe}$ with mass could also reflect a shorter formation timescale for the more massive galaxies (Worthey et al. 1992), as might perhaps be expected since current cosmological simulations suggest that the biggest galaxies tend to form first. The similar variations in the ratio of alpha-elements to iron that are seen in other old stellar populations are all well explained by the expected larger contribution of type II supernova products in older populations (McWilliam 1997), so a similar explanation may apply also to the $\mathrm{Mg} / \mathrm{Fe}$ ratio in ellipticals. The remaining trends that might still reflect a varying IMF are then the systematic increases of both mass-to-light ratio and metallicity with mass among early-type galaxies. Other explanations of these trends are also possible (e.g., Guzmán et al. 1993; see below), but we consider first whether a variable IMF of the type discussed above can account for them.

It is noteworthy that the mass-to-light ratio $M / L$ and the magnesium abundance $\mathrm{Mg} / \mathrm{H}$ both increase with mass among early-type galaxies, since this is the opposite of what would be predicted if the IMF were a simple power law with a variable slope; the mass would then mostly be in lowmass stars and $M / L$ would anti-correlate with $\mathrm{Mg} / \mathrm{H}$. However, the observed correlation can be accounted for if the IMF is bimodal and if stellar remnants produced by an early high-mass mode of star formation contribute importantly to the mass; then, if the high-mass mode is more dominant in the more massive galaxies, both $M / L$ and $\mathrm{Mg} / \mathrm{H}$ will increase with mass (L86). A model of this type has been developed by Zepf \& Silk (1996, hereafter ZS), who show that it can account not only for the increase of $M / L$ with mass among elliptical galaxies but also for the large amount of mass in heavy elements that is observed in the hot gas in clusters of galaxies. In the ZS model the heavy elements in the hot gas are assumed to be produced by the high-mass mode of star formation, suggested by these authors to be associated with merger-induced starbursts in forming elliptical galaxies.

The predictions of the ZS model do not depend strongly on the assumption that the IMF is bimodal, but only require the IMF to be more top-heavy in the more massive galaxies. This would be true also for a single-peaked IMF such as (1) if the peak mass $m_{\mathrm{p}}$ is higher in the more massive galaxies, as might occur if the more massive galaxies form earlier than the less massive ones. The total observed increase of $M / L$ with mass is about a factor of 4 , and an increase of this magnitude would be predicted if the peak mass in IMF (1) were increased from $1 \mathrm{M}_{\odot}$ to $2.5 \mathrm{M}_{\odot}$. Values of $m_{\mathrm{p}}$ in this range do not seem implausible for star formation at large redshifts, as was noted in Section 3. However, the increase in $M / L$ that can be obtained in this way is very sensitive to the assumed form of the lower IMF; with an IMF of form (2), for example, $M / L$ increases by a much smaller factor of only 1.2 for the corresponding increase in the mass scale $m_{1}$. Thus an IMF that falls off steeply at the low end is required to obtain a sufficiently large variation in $M / L$. The model of ZS satisfies this requirement by assuming that the IMF of the high-mass mode is sharply truncated below $3 \mathrm{M}_{\odot}$. 
For a given mass in remnants, a model with a single-peaked IMF predicts a similar mass of heavy elements as does the ZS model. For example, if the total mass in remnants is $9 \times 10^{12} \mathrm{M}_{\odot}$, as in the ZS model of the Coma cluster (for $h=0.65$ ), that model predicts that the mass of iron produced is about $1.2 \times 10^{11} \mathrm{M}_{\odot}$, in satisfactory agreement with the observed iron content of $7 \times 10^{10} \mathrm{M}_{\odot}$; for IMF (1) with $m_{\mathrm{p}} \sim 2 \mathrm{M}_{\odot}$, the amount of iron produced with the same stellar production rate (Woosley \& Weaver 1995) is about $8 \times 10^{10} \mathrm{M}_{\odot}$, again in satisfactory agreement with the observations. This agreement is not very sensitive to the assumed value of $m_{\mathrm{p}}$, and it shows that a smoothly peaked IMF of form (1) can account for the observed amount of heavy elements in the hot gas of the Coma cluster as well as can the bimodal IMF of ZS.

Many efforts to model in more detail the properties of elliptical galaxies and the hot gas in clusters have also concluded that the IMF in the proto-ellipticals must have been top-heavy (Elbaz, Arnaud, \& Vangioni-Flam 1995; Loewenstein \& Mushotzky 1996; Gibson 1997; Gibson \& Matteucci 1997b), and possibly more top-heavy in the more massive galaxies (Matteucci 1994; Angeletti \& Giannone 1997). Most of these studies have tried to model the chemical evolution of elliptical galaxies with specific galactic wind models, and most have assumed that the IMF is a power law with an adjustable slope, in which case a slope in the range $x \sim 0.8$ to 1.0 is favored. However, the same observations can be fitted equally well with an IMF of form (1) or (2) in which the mass scale $m_{1}$ is larger than in the standard present-day IMF. The strongest constraint on the early IMF in clusters of galaxies, which does not depend on the details of galactic evolution models, is provided by the large total mass of heavy elements observed, mostly in the hot gas. Typically, the total mass of heavy elements is a few times that predicted by standard models based on a Salpeter IMF; in the Coma cluster, for example, the ratio of heavy-element mass to total cluster luminosity is about 3 times what would be predicted for a Salpeter IMF. An increase by a factor of 3 in the amount of heavy elements produced relative to the number of low-mass stars formed is the same effect that was needed above to solve the G-dwarf problem, and it can be achieved with the same kind of change in the IMF at early times, for example by an increase in the mass scale $m_{1}$ by a factor of 4 for IMF (1). This has approximately the same effect as changing the slope $x$ of a power-law IMF by -0.4 , but an IMF of form (1) or (2) with a variable mass scale may be more consistent with the evidence mentioned in Section 2 suggesting that the IMF has a universal Salpeter-like form for large masses while it flattens or declines at the low end.

A finer point concerns the relative abundances of the various heavy elements that can now be measured in the hot gas (Mushotzky et al. 1996; Loewenstein \& Mushotzky 1996). These and previous studies have generally found that alpha-elements such as oxygen and silicon are overabundant relative to iron, as would be expected if the hot gas had been enriched mainly by type II supernovae. However, when the $\alpha / \mathrm{Fe}$ ratios are corrected for a recent downward revision of the solar iron abundance, they become only slightly higher than solar and suggest a significant contribution of iron from type Ia supernovae (Ishimaru \& Arimoto 1997). This conclusion has been questioned by Gibson, Loewenstein \& Mushotzky (1997) because of the uncertainties that remain in the predicted heavy-element yields, but $\alpha / \mathrm{Fe}$ ratios that are only slightly above solar and that reflect a significant contribution from type Ia supernovae would in fact be expected if the IMF always has a Salpeter-like slope at the upper end like IMFs (1) or (2), since the predicted ratio of type Ia to type II supernovae is then nearly the same as for a standard IMF. Note that the abundance ratios observed in the hot gas differ, as would be expected, from those in the stars, since the stars are enriched only by early supernovae that are mostly of type II, so that the stars show enhanced $\alpha / \mathrm{Fe}$ ratios, while the hot gas is enriched also by later type Ia supernovae and thus shows nearly solar abundance ratios (Renzini 1997). Loewenstein \& Mushotzky (1996) note that there is marginal evidence from some of the element ratios favoring a Salpeter slope over a smaller slope for the massive stars, and thus favoring an IMF of form (1) or (2) over a simple power law with $x \sim 0.9$ at all masses. Wyse (1997) questions the need for any nonstandard IMF and suggests that most of the cluster data are consistent with a universal Salpeter-like IMF, but 
notes nevertheless that the high overall abundances of heavy elements observed in some clusters may be difficult to reconcile with a universal IMF.

In summary, we conclude that the chemical properties of the galaxies and the hot gas in clusters can be accounted for satisfactorily if the early IMF has a form similar to (1) or (2) with a mass scale $m_{1}$ of the order of $1-2 \mathrm{M}_{\odot}$. An IMF of this form may be more consistent with the observed abundance ratios than a simple power-law IMF with $x \sim 0.9$ at all masses, which otherwise could account equally well for the overall enrichment in heavy elements. The increase of $M / L$ with mass among elliptical galaxies might also be explainable if the mass scale $m_{1}$ increases with galactic mass, but only if the IMF falls off steeply at the low end like IMF (1), i.e. more steeply than is suggested by most of the evidence concerning the IMF in our Galaxy. Thus the increase in $M / L$ with mass among elliptical galaxies is not as easily accounted for with a varying IMF as are the chemical abundances in galaxies and clusters, since more extreme assumptions are required. The alternative possibility that the increase of $M / L$ with mass results from a decrease in the spatial concentration of the visible matter relative to the dark matter in elliptical galaxies may therefore be a more promising explanation of the observed trend (Guzmán et al. 1993); as was noted by these authors, a decrease in the relative spatial concentration of the visible matter and hence an increase in the measured mass-to-light ratio with increasing galaxy mass would be expected if mergers play an increasingly important role in the formation of the more massive galaxies.

The chemical abundances in the hot gas can of course only constrain the IMF averaged over an entire cluster and not the IMF in any individual type of galaxy. The question of whether dwarf galaxies contribute importantly to the enrichment of the hot gas (Trentham 1994, Gibson \& Matteucci 1997a) is therefore not relevant to this integral constraint. The essential property of cluster galaxies causing them to have a systematically top-heavy IMF may simply be that as a group, they are dominated by very old stellar populations that probably contain the first visible stars to be formed in the universe. If the mass scale for star formation was indeed generally higher at early times, then clusters of galaxies could plausibly have had an average IMF that was systematically more top-heavy than that found, for example, in the local disk of our Galaxy or in the Magellanic Clouds.

\subsection{Energetics of the hot gas in clusters}

If the early star formation in clusters of galaxies had a top-heavy IMF, this would imply not only more heavy-element production but also more energy input to the hot gas from supernovae than is predicted by standard models. Since the total mass of heavy elements per unit luminosity in clusters is typically a few times that predicted for a Salpeter IMF, the number of supernovae that occurred at early times must also have been a few times larger than in standard models. As discussed by Elbaz et al. (1995) and Loewenstein \& Mushotzky (1996), this means that a larger amount of energy would have been available to drive galactic winds, and suggests that a substantial fraction, perhaps half, of the initial mass of the cluster galaxies could have been ejected in such winds. The fact that most of the heavy elements in clusters are in the intracluster medium rather than in the galaxies implies that the cluster galaxies indeed lost most of their supernova-enriched gas, in contrast to conventional models where only a modest fraction of the gas is lost (Larson \& Dinerstein 1975).

These energetic outflows can contribute to heating the intergalactic medium, and such extra heating may be required because the hot gas in clusters has a more extended spatial distribution than the galaxies or the dark matter and more energy per unit mass than the galaxies (David, Jones \& Forman 1995, 1996). According to these authors, the thermal energy per unit mass of the hot gas is typically about twice the kinetic energy per unit mass of the elliptical galaxies, and this implies an excess thermal energy of the order of $4 \times 10^{49}$ ergs per $\mathrm{M}_{\odot}$ of visible stars. If this energy comes from supernovae, and if the energy supplied per supernova is equal to the explosion energy of $10^{51} \mathrm{ergs}$, then one supernova is required for every $25 \mathrm{M}_{\odot}$ of visible stars, about 3 times 
the number predicted for a standard IMF. If significant supernova energy is lost by radiative cooling, as is expected (Larson 1974), then even more supernovae are needed. Thus the number of supernovae needed to heat the gas may exceed the number predicted for a standard IMF by a significant factor, again suggesting a top-heavy early IMF.

If supernovae can indeed supply a thermal energy comparable to the binding energy of the gas in a cluster of galaxies, then large amounts of gas may be lost not only from individual galaxies but also from entire groups and small clusters, and only the largest clusters (if any) may have been able to retain all of their enriched gas. Both the gas content and the heavy-element content of groups and clusters increase with cluster size (David et al. 1995), and this suggests that most groups and small clusters have indeed lost most of their gas and that only the largest ones have evolved as closed systems (David 1997; Renzini 1997). If this is true, then a significant fraction of the heavy elements ever produced in the universe may have been ejected from galaxies into a hot intergalactic medium that is not bound in clusters and has not yet been observed. Although present estimates of the history of massive star formation in the universe seem consistent with its known heavy-element content, a significant amount of early star formation could have been missed in these estimates because of the neglect of dust extinction (see Section 5); in this case a top-heavy early IMF may be required quite generally, even outside clusters, and galaxies might have produced more heavy elements than are predicted by standard models and might have ejected a significant fraction of their heavy elements into an intergalactic medium.

Thus the energetics of the hot gas in clusters suggests, in agreement with the heavy-element content, that the early supernova rate in clusters may have been at least 3 times that predicted by standard models, implying that the early IMF produced at least 3 times more massive stars per solar-mass star than a Salpeter IMF. The detailed shape of the early IMF is not constrained by this evidence, which could be accounted for equally well by a power-law IMF with $x \sim 0.9$, an IMF of form (1) or (2) with $m_{1} \sim 1.5 \mathrm{M}_{\odot}$, or a bimodal IMF. However, an IMF similar to (1) or (2) seems preferable for the reasons mentioned above, including the fact that it is more consistent with the form of the present-day IMF and the fact that it yields better consistency with the observed abundance ratios in clusters than a simple power law.

\section{LUMINOSITY EVOLUTION OF GALAXIES}

If the old stars responsible for most of the present luminosity of early-type galaxies were formed with a top-heavy IMF, these galaxies will fade more rapidly with time than would be the case with a standard IMF. According to Tinsley (1980), the luminosity of an old stellar system varies with time as $L \propto t^{-1.3+0.3 x}$; thus if $x$ changes by -0.5 , the exponent of $t$ changes by only -0.15 and the effect may be difficult to measure, while if the value of $x$ becomes as small as 0 at $m \sim \mathrm{M}_{\odot}$, as in some of the examples considered above, the exponent of $t$ changes by -0.4 and the effect may become measurable, implying an extra dimming by a factor of 2 in luminosity over a factor of 5 in cosmic time. For actively star-forming galaxies whose light is dominated by massive stars, the effect of a top-heavy IMF on luminosity evolution can be much more dramatic, especially if the star formation rate declines strongly with time; if the IMF contains relatively few low-mass stars, a system that is initially extremely luminous can then evolve into one that is extremely faint.

Direct studies of galaxy evolution by observations at a range of redshifts are still at an early stage, but the first results of such studies suggest that a relatively flat early IMF may be required to account for the data. Lilly et al. (1996) have used data from the CFRS redshift survey to determine the evolution of the comoving luminosity density of the universe since a redshift of $z=1$, and they find strong evolution that is compatible with simple models of galaxy evolution based on a Salpeter IMF but incompatible with models based on the steeper Scalo (1986) IMF. If dust extinction is important, these results may require an IMF that is even flatter than the Salpeter IMF. Totani, Yoshii \& Sato (1997) find that the Lilly et al. (1996) results are inconsistent 
with the galaxy evolution models of Arimoto \& Yoshii (1987), again based on a Salpeter-like IMF, unless a large cosmological constant is assumed, but an alternative explanation for the discrepancy would be a flatter IMF. Rapid fading due to a top-heavy IMF might also help to account for the apparent disappearance of many 'faint blue galaxies' that are abundant at intermediate redshifts but that appear to have no equally abundant counterparts at low redshift (Ellis 1997; Ferguson \& Babul 1998).

Madau et al. (1996), Guzmán et al. (1997), and Madau, Pozetti \& Dickinson (1998) have used observations of high-redshift galaxies in the Hubble Deep Field to extend the results of Lilly et al. (1996) to a redshift $z \sim 4$, and they find again that the evolution of the luminosity density of the universe at various wavelengths is compatible with models of galaxy evolution based on a Salpeter IMF but incompatible with models based on the steeper Scalo IMF, and possibly incompatible even with a Salpeter IMF if extinction is sufficiently important. The neglect of dust extinction is questionable since actively star-forming galaxies at low redshifts generally show heavy obscuration and radiate much of their energy at infrared wavelengths, and this may be true also for star-forming galaxies at larger redshifts (Mazzei \& De Zotti 1996; Rowan-Robinson et al. 1997). Smail, Ivison \& Blain (1997) have discovered an abundant class of lensed submillimeter sources at redshifts $z>1$ that could be heavily reddened young galaxies, and they suggest that a large upward revision may be needed in the cosmic rate of massive star formation at high redshifts estimated by Madau et al. (1996). In discussing the interpretation of these results, Blain et al. (1998) conclude that unless obscured AGNs account for a major fraction of the observed flux, a top-heavy early IMF is probably required in order not to produce too many low-mass stars that would remain visible to the present time.

Recently, a far-infrared background radiation has been detected in the COBE data that could come from heavily obscured, actively star-forming galaxies at high redshifts (Puget et al. 1996; Guiderdoni et al. 1997; Schlegel, Finkbeiner \& Davis 1998; Hauser et al. 1998). The background radiation intensity reported by Hauser et al. (1998) is similar to the level predicted by ZS from their model invoking an early high-mass mode of star formation to account for the abundance of heavy elements in clusters, and therefore this observation is consistent with, and may provide support for, models with a top-heavy early IMF. In discussing possible interpretations of the far-IR background, Dwek et al. (1998) show that all of the models that have been proposed to describe the cosmic history of star formation that are consistent with low-redshift observations fail by at least a factor of 2 to account for the observed background intensity, but they show that it can be accounted for with a model that includes a period of formation of exclusively massive stars at high redshifts, as was was proposed in the models of Elbaz et al. (1995) and ZS that postulate a bimodal IMF. Again, we note that the detailed form of the IMF is not constrained by this evidence, which could be accounted for equally well with an IMF similar to (1) or (2) in which the mass scale $m_{1}$ was higher at early times.

Thus, while studies of high-redshift galaxies and infrared background radiation do not yet provide conclusive evidence for time-variability of the IMF, they have the potential to test in a relatively direct way whether the global IMF has varied with time, and some recent data suggest that such variability has indeed occurred. Further observations capable constraining the cosmic history of massive star formation will therefore be of great interest for constraining the time evolution of the IMF. 


\section{DARK MATTER AND MACHOS}

A question of current interest is whether the remnants of large numbers of massive stars formed at early times could contribute significantly to the dark matter in galactic halos (Larson 1987; Burkert \& Silk 1997). Evidence that this might be the case has recently been provided by the claimed detection via gravitational microlensing of stellar-mass 'machos' in the halo of our Galaxy (Alcock et al. 1997); these authors estimate that half of the dark mass in the Galactic halo out to a radius of $50 \mathrm{kpc}$ could be in the form of machos with a typical mass of $\sim 0.5 \mathrm{M}_{\odot}$. The only known dim objects of this mass are white dwarfs, but if the estimated typical macho mass increases as microlensing events of longer duration are recorded, neutron stars and black holes might also become candidates. All of these types of objects could in principle exist as the remnants of large numbers of massive stars formed at early times in the Galactic halo. Several authors have considered the viability of such explanations of the macho results and have concluded that they are strongly constrained by the scarcity of visible low-mass halo stars and by the lack of evidence for the expected heavy-element enrichment of the Galaxy, implying an IMF that is sharply peaked at a few $\mathrm{M}_{\odot}$ and falls off very steeply at both lower and higher masses; moreover, any significant whitedwarf component must be very old in order not to conflict with observed white dwarf numbers (Adams \& Laughlin 1996; Flynn, Gould \& Bahcall 1996; Kawaler 1996; Chabrier, Segretain \& Méra 1996; Graff, Laughlin \& Freese 1998). The constraint on the upper IMF is relaxed if most of the heavy elements produced by the massive stars are ejected into an intergalactic medium, possibly by starburst activity associated with the formation of the halo, as suggested by Fields, Mathews \& Schramm (1997); these authors propose a model for halo formation with a lognormal IMF that peaks at $2.3 \mathrm{M}_{\odot}$, and they claim that it can account for $\sim 50 \%$ of the dark mass as remnants. This model is qualitatively similar to the models with a peaked IMF considered in Section 4.2 to describe the early evolution of elliptical galaxies in clusters, where there is indeed evidence that most of the heavy elements are ejected into an intergalactic medium; therefore it is of interest to consider whether similar models can account for significant amounts of dark matter as remnants.

If we consider an IMF of form (1) as a possible description of early star formation in the Galactic halo, an old population with an IMF of this form has a mass-to-light ratio of about 17 if the peak mass $m_{\mathrm{p}}$ is $2.5 \mathrm{M}_{\odot}, 120$ if $m_{\mathrm{p}}$ is $4 \mathrm{M}_{\odot}$, and 1800 if $m_{\mathrm{p}}$ is $6 \mathrm{M}_{\odot}$; the associated mass is mostly in white dwarfs for the smaller value of $m_{\mathrm{p}}$ and mostly in neutron stars and black holes for the larger values. Thus, it is possible in principle to account for large amounts of dark matter with an IMF of this form if the peak mass $m_{\mathrm{p}}$ is high enough. The predicted amount of dark mass could be even larger if metal-poor stars lose less mass in winds than metal-rich stars (Maeder 1992) and eject less mass in supernova explosions (Woosley \& Weaver 1995), leaving more mass behind in remnants. However, the possibility of achieving a very large $M / L$ in this way depends critically on having an IMF that falls off sufficiently steeply at low masses, as has already been noted (Section 4.2); with an IMF of form (2), for example, the predicted values of $M / L$ for the cases considered above are only about 6,7 , and 8 respectively. A sharp truncation of the lower IMF could of course yield an arbitrarily large $M / L$, but this does not seem plausible physically, and even the smooth exponential falloff of IMF (1) at low masses is steeper than is suggested by the available evidence concerning the local IMF. The possibility that population III stars might have had a sufficiently strongly truncated lower IMF to allow the existence of large amounts of dark matter in the remnants of such stars will be discussed in Section 7.

If the galaxies in clusters have as much associated dark matter in the form of machos as has been suggested to exist in the halo of our Galaxy (Alcock et al. 1997), such machos would make a significant contribution to the cluster mass. David (1997) has noted that if half of the dark matter associated with cluster galaxies out to a radius of $50 \mathrm{kpc}$ is in the form of stellar remnants, then the baryonic content of clusters could be as large as $\sim 50 \%$, with remnants contributing $\sim 10-20 \%$ of the total. Most of the hot gas in clusters might then have been processed through the massive 
remnant progenitors. However, the total amount of mass in remnants would then be 10-20 times larger than in the ZS model discussed in Section 4.2, in which remnants make up only about $1 \%$ of the cluster mass; the amount of heavy elements produced would therefore also be larger by a factor of 10-20 than in the ZS model, and larger by a similar factor than the amount observed, assuming the same production rates in massive stars. Thus, such a model is not viable unless nearly all of the heavy elements produced by the massive stars are either lost from the cluster, which seems unlikely, or trapped in black-hole remnants. Very massive population III stars are predicted to collapse completely into black holes (Section 7), but such black holes would be much more massive than the machos detected so far in the Galactic halo, and therefore could not account for the macho results.

We conclude, as have previous authors, that it is difficult with any plausible assumptions to construct models that can account for a large fraction of the dark matter in the universe as being in low-mass stellar remnants such as white dwarfs, neutron stars, or small black holes. Some small fraction of the dark matter, perhaps a few percent, could plausibly be in this form, but the only apparent way to account for large amounts of dark matter as stellar remnants would be with the massive remnants of very massive population III stars.

\section{POPULATION III STARS}

When the first stars formed, no heavy elements had yet been produced and recycled into new stars by supernovae, so these first 'population III' stars (Carr 1994) would have contained no heavy elements. The complete absence of heavy elements leads to qualitative differences in star formation and stellar evolution that can have important consequences for the stellar IMF and heavy-element production. Woosley \& Weaver (1995) have noted, for example, that metal-free stars develop much more compact cores than stars with finite metallicity, with the result that less of the core mass is ejected in a supernova explosion and more ends up in a black hole; this reduces the heavy-element production rate by about a factor of 4 for a standard IMF. Thus population III stars might be better candidates for dark-matter progenitors than stars with finite metallicity, both because of the predicted higher remnant masses and because of the reduced heavy-element production. However, an even more important difference may be that in the complete absence of heavy elements, star formation almost certainly produces a very different and much more top-heavy IMF (see below).

Would one expect significant numbers of population III stars to have formed? The failure of astronomers to find any metal-free stars despite many years of searching has long been a puzzle, and the explanation usually offered is that very few such stars were needed to produce the very small amount of heavy elements observed in the most metal-poor stars. However, it is not very plausible that the number of metal-free stars formed should have been negligibly small. The first stars with finite metallicity could only have appeared after the first metal-free stars had completed their evolution and exploded as supernovae, and after the resulting hot supernova ejecta had cooled, mixed with the surrounding gas, and become incorporated into new collapsing clouds and stars. The lifetimes of massive stars are at least a few million years, and the time required for their supernova ejecta to be recycled into new stars is probably at least of the same order; at present, this recycling time is of the order of tens of millions of years (Larson 1996). Thus, the time interval between the formation of the first metal-free stars and the formation of the first finite-metallicity stars was probably not a negligible fraction of the age of the universe when star formation began; at a minimum, it must have been at least $1 \%$ of the age of the universe at that time. If stars continued to form during this interval, an appreciable number of metal-free stars could have formed.

If population III stars did form in significant numbers, they must have had an IMF that contained essentially no solar-mass stars, since no metal-free solar-mass stars have yet been seen. An IMF strongly biased toward high masses would probably be expected for population III stars, since in the absence of heavy elements the only available coolant in primordial clouds is molecular 
hydrogen, which cannot cool the gas much below about $100 \mathrm{~K}$. A number of recent studies of the thermal properties of primordial gas clouds have predicted temperatures in the range $\sim 100-1000 \mathrm{~K}$ that are one to two orders of magnitude higher than the temperature in present-day star-forming clouds (Anninos \& Norman 1996; Haiman, Thoul \& Loeb 1996; Tegmark et al. 1997). The Jeans mass at a given pressure would therefore have been 2 to 4 orders of magnitude higher in such primordial clouds than in present-day star-forming clouds. If the pressures in the primordial clouds were not very much higher than those in present-day clouds, the Jeans mass for primordial star formation would then have been several orders of magnitude higher than that characterizing present-day star formation.

A further important result is the demonstration by Uehara et al. (1996) that if molecular hydrogen is the only coolant, the minimum fragment mass that can be attained before high opacity to the cooling radiation prevents further fragmentation is approximately the Chandrasekhar mass. From a more detailed calculation, Nakamura \& Umemura (1998) find that this minimum mass is about $4 \mathrm{M}_{\odot}$, and they suggest that most population III stars formed with masses larger than $16 \mathrm{M}_{\odot}$. The minimum fragment mass set by opacity is much smaller than the Jeans mass, and it is a strong lower limit that is not sensitive to details of the star formation process such as the geometry of the fragmenting cloud. Under present conditions, this lower limit is about $0.007 \mathrm{M}_{\odot}$ (Low \& Lynden-Bell 1976; Rees 1976; Silk 1977), but few if any stellar objects with such a small mass have been found, and the great majority of stars have much larger masses. Thus, the opacity limit may in practice rarely be reached, and most stars may form with much larger masses that are closer to the Jeans mass. These considerations suggest that both the characteristic mass of the IMF and the minimum stellar mass were shifted upwards by several orders of magnitude for population III stars; most of these stars might then have had masses of hundreds or thousands of solar masses, while none had masses as small as $1 \mathrm{M}_{\odot}$. Thus, significant numbers of massive population III stars could have existed without conflicting with the observed absence of solar-mass metal-free stars.

Can the remnants of population III stars contribute importantly to the dark matter in the universe? For this to be possible, several strong requirements must be satisfied: (1) Early star formation must have been very efficient, and must have processed most of the baryonic content of the universe into massive stars. Present-day star formation is very inefficient, but the first star formation might have been more efficient if the negative feedback effects that now limit the rate of star formation were less important; radiation pressure and stellar winds, for example, might have been much less important in the absence of heavy elements. (2) If most of the stellar mass is to be converted into dark matter, these massive stars must then collapse with high efficiency into dark objects, presumably mostly black holes. Moreover, if heavy elements are not to be greatly overproduced, most of the heavy elements synthesized in these stars must also end up in black holes. These requirements might be satisfied for 'very massive objects' with masses larger than $200 \mathrm{M}_{\odot}$, which are predicted to collapse completely into black holes (Carr 1994). Another possibility might be that some massive stars undergo secular core contraction to a black hole and thus 'fizzle' without producing a supernova event (Hayashi, Eriguchi \& Hashimoto 1998). (3) If limits on the cosmic background radiation at various wavelengths are not to be exceeded, the formation of large numbers of massive population III stars is constrained to occur at very large redshifts of the order of 30 or more (Carr 1994). Currently popular variants of standard CDM cosmology do not predict large amounts of star formation at such high redshifts, but significant star formation might occur at such redshifts in some less conventional cosmological models that have been proposed, such as a purely baryonic universe (Peebles 1987, 1993; Gnedin \& Ostriker 1992; Cen, Ostriker \& Peebles 1993).

Given the number of requirements that must be satisfied, it does not presently seem very likely that the remnants of massive population III stars can be major contributors to the dark matter. However, even if they do not contribute importantly to explaining machos or dark matter, population III stars might still play an important role in pre-enriching the gas in galaxies and 
helping to solve the ubiquitous G-dwarf problem. If such stars produce enough oxygen to preenrich the gas in the disk of our Galaxy to half of the present median stellar oxygen abundance, then the total mass left behind in their remnants, assuming a standard upper IMF and the oxygen production rates of Woosley \& Weaver (1995) for metal-free stars, will be about $5 \times 10^{9} \mathrm{M}_{\odot}$, which is a small fraction of the mass of the Galactic disk and an even smaller fraction of the halo mass. Thus the predicted amount of mass in remnants does not rule out the possible existence of enough population III stars to solve the G-dwarf problem. Since a metal-free early population should contain no stars with masses of a solar mass or less, there would also be no conflict with the lack of evidence for a non-standard IMF among the stars in the Galactic halo and elsewhere. The only apparent constraint on, and evidence for, an early population of metal-free stars would then be any distinctive abundance patterns that might be left behind in their nucleosynthetic products. Unusual abundance patterns are indeed observed in some of the most metal-poor stars (McWilliam 1997), and some of them could result from enrichment by population III stars; for example, Norris, Ryan \& Beers (1997) suggest that the high carbon-to-iron ratios that they find in many very metalpoor stars with $[\mathrm{Fe} / \mathrm{H}]<-2.5$ could reflect enrichment by metal-free stars with masses of about 20-30 $\mathrm{M}_{\odot}$, which according to Woosley \& Weaver (1995) are predicted to yield high carbon-to-iron ratios.

We conclude that population III stars could play a role in solving the G-dwarf problem in galaxies, and perhaps also in accounting for the anomalous abundance ratios that have been seen in some of the most metal-poor stars. Population III stars might also account for the reionization of the universe that is needed to explain the highly ionized state of the intergalactic medium (Haiman \& Loeb 1997). However, once again, it does not seem likely that large amounts of dark matter can be accounted for by the remnants of such stars; this would be possible only if large numbers of such stars were formed with high efficiency at very high redshifts, which is not predicted by current cosmological models. Population III stars may thus be most important for their effects on the energetics and chemical enrichment of the early universe.

\section{CONCLUSIONS}

In the absence of any clear direct evidence for a time-varying IMF, the possible existence of a top-heavy IMF at early times remains hypothetical. Some of the more extreme changes in the IMF that have been proposed to explain various observations can probably now be ruled out, but a number of lines of evidence continue to suggest at least moderate variability of the IMF with time. The longest-standing problem in the theory of galactic chemical evolution, the G-dwarf problem, persists and now even seems ubiquitous, suggesting that its solution may be a general phenomenon such as a time-varying IMF, as was discussed in Section 4.1. The amount of variability in the IMF that is required to solve the G-dwarf problem is moderate, amounting to an increase by only a factor of $\sim 3$ in the ratio of high-mass to low-mass stars formed during the early stages of galactic evolution; this kind of variation is not excluded by any of the constraints that have been discussed.

The strongest evidence for variability of the IMF, with which most authors concur, is the large total mass of heavy elements observed in clusters of galaxies, mostly in the hot medium; the change in the IMF needed to account for these observations is again moderate, typically involving an increase by a factor of $\sim 2-3$ in the ratio of high-mass to low-mass stars formed during the early stages of cluster evolution (Section 4.2). The energetics of the hot gas may also provide evidence that more supernovae were produced at early times than would be predicted by a standard IMF (Section 4.3). All of this evidence could be accounted for if the slope $x$ of a power-law IMF were reduced by about 0.4 , or if the mass scale $m_{1}$ in an IMF of form (1) or (2) were increased by about a factor of 4 in young cluster galaxies. The latter type of variability is not implausible, as was discussed in Section 3, and the steeper slopes of IMFs (1) and (2) at large masses yield better 
agreement with the observed abundance ratios in clusters than does a simple power law with a shallow slope at all masses (Section 4.2).

Somewhat more direct evidence for time-variability of the IMF may be provided by studies of high-redshift galaxies and by observations of infrared background radiation that suggest high rates of massive star formation at early times; these observations may require a top-heavy early IMF in order not to predict too many low-mass stars that would remain visible to the present time (Section 5). The amount of variation in the IMF that may be needed to account for these observations is again of the same order as that required to account for the heavy-element content of clusters of galaxies. Thus, there appears to be a significant body of evidence emerging that is consistent with the kind of moderate variability of the IMF with time that has been discussed in this paper. Clearly, however, all of the evidence and constraints that have been discussed here need to be strengthened before firm conclusions can be drawn.

Similar moderate variability of the IMF cannot, however, produce large variations in the massto-light ratios of galaxies or account for large amounts of dark matter in the form of stellar remnants; this requires more extreme assumptions about the form of the early IMF, which must be strongly truncated at the lower end (Section 6.). Metal-free 'population III' stars might have had such a strongly truncated lower IMF, since the lower mass limit for such stars is predicted to be above a solar mass (Section 7.). Thus, massive population III stars could have existed in significant numbers without conflicting with the absence of solar-mass metal-free stars, and they might help to account for some of the evidence that has been attributed to a top-heavy early IMF. In principle they could also contribute significantly to cosmic dark matter by the production of massive black-hole remnants, but even if they do not, they can still contribute importantly to the energetics and chemical enrichment of the early universe. Further study of the processes of early star formation and stellar evolution will thus be of great importance for developing a better astrophysical understanding of the early universe and of the origin of the stars and galaxies that we now see.

\section{REFERENCES}

Adams F. C., Laughlin, G., 1996, ApJ, 468, 586

Alcock C., et al. (the MACHO collaboration), 1997, ApJ, 486, 697; see also ApJ, 490, L59 and ApJ, 491, L11

Angeletti L., Giannone, P., 1997, A\&A, 321, 343

Anninos P., Norman M. L., 1996, ApJ, 460, 556

Arimoto N., Yoshii Y., 1987, A\&A, 173, 23

Basri G., Marcy G. W., 1997, in Holt S. S., Mundy L. G., eds., Star Formation Near and Far. AIP, Woodbury, NY, p. 228

Blain A. W., Smail I., Ivison R. J. \& Kneib J.-P., 1998, MNRAS, in press (astro-ph/9806062)

Bressan A., Chiosi C., Fagotto F., 1994, ApJS, 94, 63

Burkert A., Silk J., 1997, ApJ, 488, L55

Burstein D., Bender R., Faber, S. M., Nolthenius, R., 1997, AJ, 114, 1365

Carr B., 1994, ARA\&A, 32, 531

Cen R., Ostriker J. P., Peebles P. J. E., 1993, ApJ, 415, 423

Chabrier G., Méra D., 1997, A\&A, 328, 83

Chabrier G., Segretain L., Méra D., 1996, ApJ, 468, L21

Dahn C. C., Liebert J., Harris H. C., Guetter H. H., 1995, in Tinney C. G., ed., The Bottom of the Main Sequence and Beyond. Springer-Verlag, Berlin, p. 239

David L. P., 1997, ApJ, 484, L11

David L. P., Jones C., Forman W., 1995, ApJ, 445, 578

David L. P., Jones C., Forman W., 1996, ApJ, 473, 692 
Dwek E., Arendt R. G., Hauser M. G., Fixsen D., Kelsall T., Leisawitz D., Pei Y. C., Wright E. L., Mather J. C., Moseley S. H., Odegard N., Shafer R., Silverberg R. F., Weiland J. L., 1998, ApJ, in press (astro-ph/9806129)

Elbaz D., Arnaud M., Vangioni-Flam E., 1995, A\&A, 303, 345

Ellis R. S., 1997, ARA\&A, 35, 389

Elmegreen B. G., 1997, ApJ, 486, 944

Faber S. M., 1977, in Tinsley B. M., Larson R. B., eds., The Evolution of Galaxies and Stellar Populations. Yale University Observatory, New Haven, p. 157

Ferguson H. C., Babul A., 1998, MNRAS, 296, 585

Festin L., 1998, A\&A, 333, 497

Fields B. D., Mathews G. J., Schramm D. N., 1997, ApJ, 483, 625

Flynn C., Gould A., Bahcall J. N., 1996, ApJ, 466, L55

Friel, E. D., 1995, ARA\&A, 33, 381

Gibson B. K., 1997, MNRAS, 290, 471

Gibson B. K., Matteucci F., 1997a, ApJ, 475, 47

Gibson B. K., Matteucci F., 1997b, MNRAS, 291, L8

Gibson B. K., Loewenstein M., Mushotzky R. F., 1997, MNRAS, 290, 623

Gnedin N. Y., Ostriker J. P., 1992, ApJ, 400, 1

Goodman A. A., Barranco J. A., Wilner D. J., Heyer M. H., 1998, ApJ, 504, in press

Graff D. S., Laughlin G., Freese K., 1998, ApJ, 499, 7

Guiderdoni B., Bouchet F. R., Puget J.-L., Lagache G., Hivon E., 1997, Nature, 390, 257

Guzmán R., Lucey J. R., Bower R. G., 1993, MNRAS, 265, 731

Guzmán R., Gallego J., Koo D. C., Phillips A. C., Lowenthal J. D., Faber S. M., Illingworth G. D., Vogt N. P., 1997, ApJ, 489, 559

Haiman Z., Loeb A., 1997, ApJ, 483, 21

Haiman Z., Thoul A. A., Loeb A., 1996, ApJ, 464, 523

Hayashi A., Eriguchi Y., Hashimoto M., 1998, ApJ, 492, 286

Hauser M. G., Arendt R. G., Kelsall T., Dwek E., Odegard N., Weiland J. L., Freundreich H. T., Reach W. T., Silverberg R. F., Moseley S. H., Pei Y. C., Lubin P., Mather J. C., Shafer R. A., Smoot G. F., Weiss R., Wilkinson D. T., Wright E. L., 1998, ApJ, in press (astro-ph/9806167) Hillenbrand L. A., 1997, AJ, 113, 1733

Hunter D. A., Light R. M., Holtzman J. A., Lynds R., O’Neil E. J., Grillmair C. J., 1997, ApJ, 478,124

Ishimaru Y., Arimoto N., 1997, PASJ, 49, 1

Jørgensen I., Franx M., Kjaergaard P., 1996, MNRAS, 280, 167

Kawaler S. D., 1996, ApJ, 467, L61

Kuijken K., Gilmore G., 1991, ApJ, 367, L9

Larson R. B., 1972, Nature, 236, 21 and Nature Phys. Sci., 236, 7

Larson R. B., 1974, MNRAS, 169, 229

Larson R. B., 1985, MNRAS, 214, 379

Larson R. B., 1986a, MNRAS, 218, 409 (L86)

Larson R. B., 1986b, in Norman C. A., Renzini A., Tosi M., eds., Stellar Populations. Cambridge Univ. Press, Cambridge, p. 101

Larson R. B., 1987, Comments Astrophys., 11, 273

Larson R. B., 1992a, MNRAS, 256, 641

Larson R. B., 1992b, in Tenorio-Tagle G., Prieto M., Sánchez F., eds., Star Formation in Stellar Systems. Cambridge Univ. Press, Cambridge, p. 125

Larson R. B., 1995, MNRAS, 272, 213

Larson R. B., 1996, in Kunth D., Guiderdoni B., Heydari-Malayeri M., Thuan T. X., eds., The Interplay Between Massive Star Formation, the ISM and Galaxy Evolution. Editions Frontières, Gif sur Yvette, p. 3 
Larson R. B., 1998, in Burkert A., McCaughrean M. J., eds., The Orion Complex Revisited. ASP, San Francisco, in press

Larson R. B., Dinerstein H. L., 1975, PASP, 87, 911

Lilly S. J., Le Fèvre O., Hammer F., Crampton D., 1996, ApJ, 460, L1

Loewenstein M., Mushotzky R. F., 1996, ApJ, 466, 695

Low C., Lynden-Bell D., 1976, MNRAS, 176, 367

Madau P., Ferguson H. C., Dickinson M. E., Giavalisco M., Steidel C. C, Fruchter A., 1996, MNRAS, 283, 1388

Madau P., Pozzetti L., Dickinson M., 1998, ApJ, 498, 106

Maeder A., 1992, A\&A, 264, 105; erratum in A\&A, 268, 833

Martín E. L., Zapatero Osorio M. R., Rebolo R., 1998, in Rebolo R., Martín E. L., Zapatero Osorio M. R., eds., Brown Dwarfs and Extrasolar Planets. ASP, San Francisco, p. 507

Massey P., 1998, in Gilmore G., Parry I., Ryan S., eds., The Stellar Initial Mass Function. Proceedings of the 38th Herstmonceux Conference, in press

Matteucci F., 1994, A\&A, 288, 57

Mayor M., Queloz D., Udry S., 1998, in Rebolo R., Martín E. L., Zapatero Osorio M. R., eds., Brown Dwarfs and Extrasolar Planets. ASP, San Francisco, p. 140

Mazzei P., De Zotti G., 1996, MNRAS, 279, 535

McWilliam A., 1997, ARA\&A, 35, 503

Méndez R. A., Minniti D., De Marchi G., Baker A., Couch W. J., 1996, MNRAS, 283, 666

Meusinger H., 1989, Astron. Nachr., 310, 29 and 115

Miller G. E., Scalo J. M., 1979, ApJS, 41, 513

Mirabel I. F., 1989, in Tenorio-Tagle G., Moles M., Melnick J., eds., IAU Colloq. 120, Structure and Dynamics of the Interstellar Medium. Springer-Verlag, Berlin, p. 396

Mushotzky R., Loewenstein M., Arnaud K. A., Tamura T., Fukazawa Y., Matsushita K., Kikuchi K., Hatsukade I., 1996, ApJ, 466, 686

Nakamura F., Umemura M., 1998, preprint

Norris J. E., Ryan S. G., Beers T. C., 1997, ApJ, 488, 350

Oort J. H., 1970, A\&A, 7, 381

Pagel B. E. J., 1989, in Beckman J. E., Pagel B. E. J., eds., Evolutionary Phenomena in Galaxies, Cambridge Univ. Press, Cambridge, p. 201

Peebles P. J. E., 1987, ApJ, 315, L73

Peebles P. J. E., 1993, Principles of Physical Cosmology, Princeton Univ. Press, Princeton, p. 655

Puget J.-L., Abergel A., Bernard J.-P., Boulanger F., Burton W. B., Désert F.-X., Hartmann D., 1996, A\&A, 308, L5

Rees M. J., 1976, MNRAS, 176, 483

Reid I. N., Yan L., Majewski S., Thompson I., Smail I., 1996, AJ, 112, 1472

Renzini A., 1997, ApJ, 488, 35

Richer H. B., Fahlman G. G., 1997, in Holt S. S., Mundy L. G., eds., Star Formation Near and Far. AIP, Woodbury, NY, p. 357

Rocha-Pinto H. J., Maciel W. J., 1996, MNRAS, 279, 447

Rowan-Robinson M., Mann R. G., Oliver S. J., Efstathiou A., Eaton N., Goldschmidt P., Mobasher B., Serjeant S. B. G., Sumner T. J., Danese L., Elbaz D., Franceschini A., Egami E., Kontizas M., Lawrence A., McMahon R., Norgaard-Nielsen H. U., Perez-Fournon I., Gonzalez-Serrano J. I., 1997, MNRAS, 289, 490

Salpeter E. E., 1955, ApJ, 121, 161

Scalo J. M., 1986, Fundam. Cosmic Phys., 11, 1

Scalo J., 1998, in Gilmore G., Parry I., Ryan S., eds., The Stellar Initial Mass Function. Proceedings of the 38th Herstmonceux Conference, in press (astro-ph 9712317)

Schlegel D. J., Finkbeiner D. P., Davis M., 1998, ApJ, 500, 525

Schmidt M., 1963, ApJ, 137, 758 
Schwarzschild M., Spitzer L., 1953, Observatory, 73, 77

Silk J., 1977, ApJ, 214, 152

Simon M., 1997, ApJ, 482, L81

Sommer-Larsen J., 1991, MNRAS, 249, 368

Smail I., Ivison R. J., Blain A. W., 1997, ApJ, 490, L5

Tegmark M., Silk J., Rees M. J., Blanchard A., Abel T., Palla F., 1997, ApJ, 474, 1

Tinsley B. M., 1980, Fundam. Cosmic Phys., 5, 287

Totani T., Yoshii Y., Sato K., 1997, ApJ, 483, L75

Trentham N., 1994, Nature, 372, 157

Truran J. W., Cameron A. G. W., 1971, Astrophys. Space Sci., 14, 179

Uehara H., Susa H., Nishi R., Yamada M., Nakamura T., 1996, ApJ, 473, L95

van den Bergh S., 1962, AJ, 67, 486

Vazdekis A., Casuso E., Peletier R. F., Beckman J. E., 1996, ApJS, 106, 307

von Hippel T., Gilmore G., Tanvir N., Robinson D., Jones D. H. P., 1996, AJ, 112, 192

Wakker B. P., van Woerden H., 1997, ARA\&A, 35, 217

Woosley S. E., Weaver T. A., 1995, ApJS, 101, 181

Worthey G., Dorman B., Jones L. A., 1996, AJ, 112, 948

Worthey G., Faber S. M., González J. J., 1992, ApJ, 398, 69

Wyse R. F. G., 1997, ApJ, 490, L69

Wyse R. F. G., Gilmore G., 1995, AJ, 110, 2771

Zepf S. E., Silk J., 1996, ApJ, 466, 114 (ZS) 